\title{
METAIS PESADOS DO SOLO APÓS APLICAÇÃO DE BIOSSÓLIDO. II - DISPONIBILIDADE ${ }^{(1)}$
}

\author{
M. R. BORGES ${ }^{(2)} \&$ E. L. M. COUTINHO(3)
}

\begin{abstract}
RESUMO
A utilização agrícola de resíduos industriais e urbanos com elevados teores de metais pesados tem aumentado a preocupação em determinar quais as concentrações "disponíveis" destes elementos no solo. Neste sentido, foi realizado um experimento, em casa de vegetação da FCAV-UNESP, com objetivo de comparar métodos de análise (DTPA, HCl 0,1 mol L-1, Mehlich-1 e Mehlich-3) para avaliação da disponi bilidade de $\mathrm{Cu}, \mathrm{Mn}, \mathrm{Ni}$, Pb e Zn para plantas de milho (Zea mays L.), cultivadas em dois solos que receberam biossólido e corretivos. 0 delineamento experimental utilizado foi inteiramente casualizado, com três repetições, segundo o esquema fatorial $2 \times 2 \times 5$ (dois solos, presença ou ausência de corretivos e cinco doses de biossólido). Os solos utilizados foram: Neossolo Quartzarênico órtico típico (RQ) e Latossolo Vermel ho eutroférrico argiloso (LV). Nos tratamentos com calagem, foram adicionados materiais corretivos para elevar o $\mathrm{pH}\left(\mathrm{CaCl}_{2}\right)$ do solo a 5,3. As doses de biossólido utilizadas foram de 0; 13; 26; 52 e $78 \mathrm{~g} \mathrm{vaso}^{-1}$ (equivalentes a 0; 10; 20; 40 e $60 \mathrm{t} \mathrm{ha}^{-1}$ ), com base no material seco. Nenhum dos extratores testados se destacou para avaliação conjunta de todos os metais. A inclusão do pH do solo nos modelos de regressão melhorou signi ficati vamente a relação entre o Mn acumulado na parte aérea das plantas e o determinado pelos extratores.
\end{abstract}

Termos de indexação: micronutrientes, extratores, lodo de esgoto, Latossolo, Neossolo.

(1) Parte da Tese de Mestrado do primei ro autor, apresentada à Faculdade de Ciências Agronômicas e Veterinária - FCAV/UNESP Recebido para publicação em abril de 2003 e aprovado em março de 2004.

(2) Doutorando do Programa de Pós-Graduação em Modelagem Computacional, IPRJ /UERJ . Rua Alberto Rangel s/n, Caixa Postal 97282, CEP 28630-050 Nova Friburgo (RJ ). Bolsista da CAPES. E-mail: mrborges@iprj.uerj.br

(3) Professor Titular do Departamento de Solos e Adubos, Faculdade de Ciências Agronômicas e Veterinária - FCAV/UNESP. Campus de J aboticabal. E-mail: coutinho@fcav.unesp.br 


\title{
SUMMARY: HEAVY METALS IN SOIL AFTER SEWAGE SLUDGE APPLICATION. II - AVAILABILITY
}

\begin{abstract}
The use of industrial and city wastes with high heavy metal contents in agriculture have increased the need for obtaining the concentration of such elements that areavailable in the soil. Thus, a greenhouse experiment was carried out at the FCAV-UNESP, in J aboticabal, São Paulo State, Brazil in order to compare analysis methods (DTPA, $\mathrm{HCl}$ 0.1 mol L-1, Mehlich-1, and Mehlich-3) to evaluate the $\mathrm{Cu}, \mathrm{Mn}, \mathrm{Ni}, \mathrm{Pb}$, and $\mathrm{Zn}$ availability for corn (Zea mays L.) plants in two soils (an Eutrustox and a UItipsamment). A complete randomized design with threereplications of treatments in a $2 \times 2 \times 5$ factorial arrangement (two soils, presence or absence of lime, and fivesewagesludgerates) was used. In thelimed treatments thesoi Is wereamended with agricultural limeto raisethesoil pH $\left(\mathrm{CaCl}_{2}\right)$ to 5.3. The sewage sludge rates (dry weight basis) were equi valent to $0,10,20,40$, and $60 \mathrm{t} \mathrm{ha}^{-1}$. All theextractants tested proved inefficient to evaluateavai labi lity of all metal s concurrently. Theinclusion of thesoil pH in theregression model s significantly improved therelationship between theamounts of $\mathrm{Mn}$ accumulated in the shoot and thoseextracted with DTPA, $\mathrm{HCl}$ $0.1 \mathrm{~mol} \mathrm{~L}^{-1}$, Mehlich-1, and Mehlich-3.
\end{abstract}

Index terms: micronutrients, extractant, sewage sludge, Eutrustox, Ultipsamment.

\section{INTRODUÇÃO}

Com a finalidade deminimizar a poluição dos rios, tem-se feito o tratamento dos efluentes domésticos e industriais, cujo produto final é denominado biossólido. O seu manuseio e disposição final são as fases mais preocupantes do processo.

O uso agrícola do biossólido, como fonte de nutrientes e matéria orgânica, é um método alternativo de disposição final desse resíduo, no qual se pode obter benefícios (Berton et al., 1989; Silva et al., 1998). Entretanto, a presença de metais pesados restringe sua aplicação no solo, à medida que o acúmulo desses elementos pode causar maior absorção pelas plantas (Chang et al., 1992) e provocar sua entrada na cadeia alimentar. Portanto, tornase necessário determinar as concentrações disponíveis desses el ementos no solo.

Várias soluções extratoras têm si do testadas para avaliar a disponibilidade de metais pesados no solo, principalmente daqueles que são nutrientes de plantas (Bataglia \& Raij, 1989; Mulchi et al., 1991). No entanto, tais estudos são ainda incipientes, e os resultados encontrados na literatura são bastante controversos. Mulchi et al. (1991) encontraram coeficientes de correlação significativos entreos teores de $\mathrm{Cd}, \mathrm{Cu}, \mathrm{Ni}$ e $\mathrm{Zn}$ em plantas de tabaco e aqueles extraídos pelas sol uções Mehlich-1, Mehlich-3 e DTPA de dois solos que receberam biossólido; entretanto, nenhum dos extratores foi eficiente para avaliar o Fe e o Pb. Abreu et al. (1995) concluíram que os métodos de M ehlich-1, Mehlich-3 eDTPA não foram eficientes para avaliar a disponibilidade de $\mathrm{Pb}, \mathrm{Cd}$, $\mathrm{Cr}$ e Ni, para plantas de trigo, e de $\mathrm{Pb}$ e Cd, para o feijoeiro, em 31 amostras de solos representativos do estado de São Paulo. Segundo esses autores, a inclusão de outros atributos do solo, tais como teor de argila para o $\mathrm{Cr}$ e pH para o $\mathrm{Ni}$, como variáveis independentes no model o de regressão, aumentou os coeficientes de determinação.

O obj etivo deste trabal ho foi comparar soluções extratoras (DTPA, $\mathrm{HCl}$, Mehlich-1 e Mehlich-3) para avaliação da disponibilidade de $\mathrm{Cu}, \mathrm{Mn}, \mathrm{Ni}, \mathrm{Pb}$ e Zn para plantas de milho (Zea mays L.), cultivadas em dois solos que receberam doses de biossólido e materiais corretivos.

\section{MATERIAL E MÉTODOS}

O experimento foi real izado em casa de vegetação da FCAV-UNESP, campus deJ aboticabal. Os sol os utilizados foram: Neossolo Quartzarênico (RQ) e L atossolo Vermel ho (LV) e o biossólido foi obtido na estação de tratamento da SABESP, no município de Franca (SP).

O delineamento experimental utilizado foi inteiramente casualizado, com três repetições, seguindo um esquema fatorial $2 \times 2 \times 5$ (dois solos, presença e ausência de calcário e cinco doses de biossólido), totalizando sessenta vasos. Foram cultivadas plantas de milho (Zea mays L.) para determinação da produção de matéria seca e dos teores de metais no material vegetal.

Uma descrição detalhada dos tratamentos utilizados e a caracterização química dos solos e do biossólido encontram-se na parte I deste trabalho (Borges \& Coutinho, 2004).

Após o período de incubação, amostras do solo de cada vaso, previamente secas ao ar e passadas em peneira com abertura de mal ha de $2 \mathrm{~mm}$, foram utilizadas para as extrações dos metais pesados, 
utilizando as soluções extratoras DTPA (Lindsay \& Norvell, 1978), $\mathrm{HCl} \mathrm{0,1} \mathrm{mol} \mathrm{L-1} \mathrm{(Wear} \mathrm{\&} \mathrm{Sommer,}$ 1947), Mehlich-1 (Wear \& Evans, 1968) eM ehlich-3 (Mehlich, 1984). Emtodos os métodos, após a agitação, as suspensões foram passadas por papel de filtro Whatman $n^{\circ} 42$, para a obtenção dos extratos. Os teores de metais pesados foram determinados por espectrofotometria de absor ção atômica (EAA).

Nas amostras de solo, foram determinados também o pH $\left(\mathrm{CaCl}_{2}\right)$ e os teores de matéria orgânica no solo (Raij et al., 1987).

Trinta dias após a emergência, a parteaérea das plantas de milho foi cortada rente ao solo, lavada e seca em estufa com circulação forçada de ar a $65^{\circ} \mathrm{C}$ atéatingir massa constante. O material vegetal seco foi pesado para determinação da produção de matéria seca, moído, e o teor de metais pesados foi analisado por meio de digestão nitricoperclórica, segundo Bataglia et al. (1983), e a determinação da concentração dos metais efetuada em EAA.

As análises de variância e as regressões (simples e múltiplas) foram realizadas utilizando o programa SAS (1989). As análises de regressão múltipla das quantidades acumuladas dos metais nas plantas de acordo com os teores desses no solo, pelas quatro soluções extratoras e o pH, foram realizadas testando-sea significância $(p<0,05)$ da entrada das variáveis independentes.

\section{RESULTADOS E DISCUSSÃO}

\section{Teor de matéria orgânica e pH do solo}

A adição de biossólido promoveu alterações em alguns atributos químicos dos solos. Ocorreram aumentos lineares dos teores de matéria orgânica nos dois sol os (Figura 1 ), graças ao teor de material orgânico presenteno biossólido (Borges \& Coutinho, 2004).

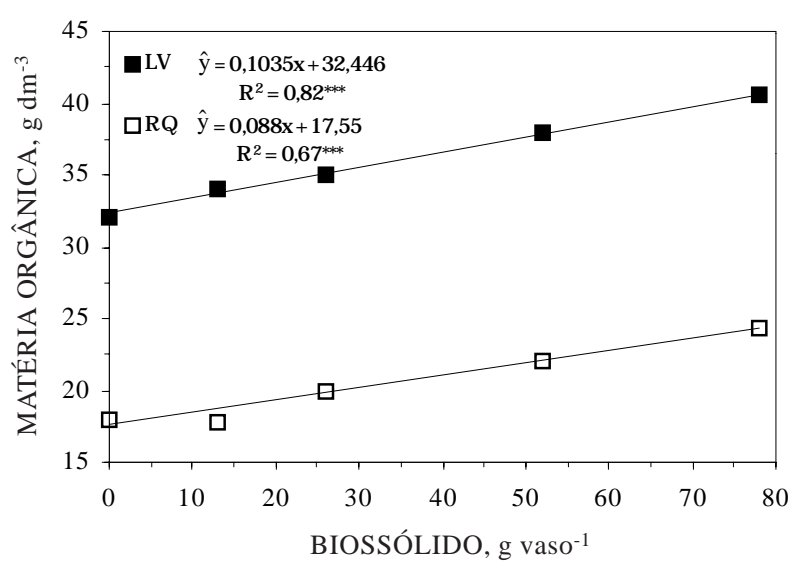

Figura 1. Teores de matéria orgânica no solo, considerando a aplicação de biossólido.
Foram verificados aumentos significativos para os valores de $\mathrm{pH}$ nos dois solos, sem corretivos (Figura 2). A el evação do pH do solo pode ter sido causada pela liberação de amônia proveniente da decomposição de compostos orgâni cos presentes no resíduo (Lund \& Doss, 1980; Berton et al., 1989).

$\mathrm{Na}$ presença de corretivos, o pH deambos os sol os manteve-se praticamente inalterado (Figura 2). Tal comportamento pode estar relacionado com o aumento da capacidadetampão do solo, proporcionado pela calagem (Lund \& Doss, 1980). Berton et al. (1997) também observaram que, na presença de corretivos, a variação nos valores de pH, causada pela aplicação de biossólido, foi muito pequena ou nenhuma.

\section{Extratores para avaliação dos metais pesados "disponíveis" no solo}

O acúmulo dos metais na parteaérea das plantas de milho (Quadro 1) foi utilizado para avaliar a eficiência das soluções DTPA, $\mathrm{HCl} 0,1 \mathrm{~mol} \mathrm{~L}^{-1}$, Mehlich-1 e Mehlich-3 em predizer a disponibilidade de $\mathrm{Cu}, \mathrm{Mn}, \mathrm{Pb}$ e $\mathrm{Zn}$ no solo, após aplicação de biossólido.

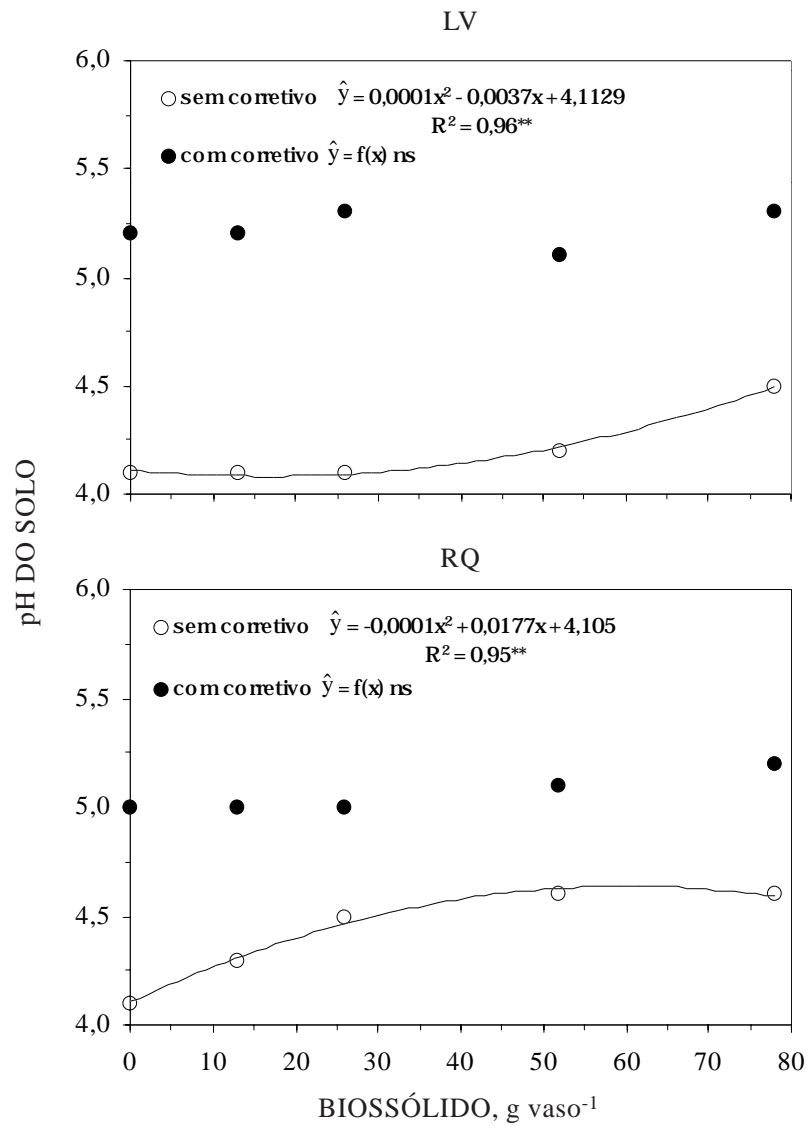

Figura 2. Valores de pH do solo, considerando a aplicação de biossólido. 
Quadro 1. Quantidade acumulada dos metais na parte aérea das plantas de milho

\begin{tabular}{|c|c|c|c|c|}
\hline \multirow{2}{*}{ Dose de biossólido } & \multicolumn{2}{|c|}{ LV } & \multicolumn{2}{|c|}{$\mathbf{R Q}$} \\
\hline & Sem corretivo & Com corretivo & Sem corretivo & Com corretivo \\
\hline \multirow[t]{2}{*}{ g vaso-1 } & \multicolumn{4}{|c|}{$-\mu \mathrm{g}$ vaso $^{-1}$} \\
\hline & \multicolumn{4}{|c|}{ Cobre } \\
\hline 0 & 25,0 & 31,0 & 11,0 & 12,0 \\
\hline 13 & 29,0 & 48,0 & 22,0 & 29,0 \\
\hline 26 & 41,0 & 50,0 & 45,0 & 47,0 \\
\hline 52 & 71,0 & 70,0 & 79,0 & 65,0 \\
\hline 78 & 108,0 & 89,0 & 89,0 & 77,0 \\
\hline$L^{(1)}$ & $* * *$ & $* * *$ & $* * *$ & $* *$ \\
\hline \multirow[t]{2}{*}{$\mathrm{Q}^{(2)}$} & ns & ns & ns & $* * *$ \\
\hline & \multicolumn{4}{|c|}{ Manganês } \\
\hline 0 & $3.841,0$ & 550,0 & 399,0 & 175,0 \\
\hline 13 & $5.689,0$ & 980,0 & 672,0 & 551,0 \\
\hline 26 & $8.449,0$ & $1.244,0$ & 940,0 & $1.331,0$ \\
\hline 52 & $8.326,0$ & $3.690,0$ & $1.036,0$ & $1.865,0$ \\
\hline 78 & $5.465,0$ & $5.960,0$ & $1.863,0$ & $2.223,0$ \\
\hline $\mathrm{L}$ & ns & ns & $* * *$ & ns \\
\hline \multirow[t]{2}{*}{$\mathrm{Q}$} & $* * *$ & $* * *$ & ns & $* * *$ \\
\hline & \multicolumn{4}{|c|}{ Níquel } \\
\hline 0 & 6,0 & 8,0 & 1,0 & 4,0 \\
\hline 13 & 5,0 & 13,0 & 2,0 & 10,0 \\
\hline 26 & 4,0 & 8,0 & 1,0 & 11,0 \\
\hline 52 & 7,0 & 8,0 & 21,0 & 18,0 \\
\hline 78 & 13,0 & 9,0 & 22,0 & 22,0 \\
\hline $\mathrm{L}$ & ns & ns & $*$ & $* * *$ \\
\hline \multirow[t]{2}{*}{ Q } & $* * *$ & ns & ns & ns \\
\hline & \multicolumn{4}{|c|}{ Chumbo } \\
\hline 0 & nd & nd & 2,3 & 1,2 \\
\hline 13 & nd & nd & 4,2 & nd \\
\hline 26 & nd & 32,0 & 2,6 & nd \\
\hline 52 & nd & 46,2 & nd & nd \\
\hline 78 & nd & 55,1 & nd & nd \\
\hline $\mathrm{L}$ & - & $* *$ & ns & - \\
\hline \multirow[t]{2}{*}{$\mathrm{Q}$} & - & ns & ns & - \\
\hline & \multicolumn{4}{|c|}{ Zinco } \\
\hline 0 & 95,0 & 116,0 & 82,0 & 88,0 \\
\hline 13 & 231,0 & 293,0 & 651,0 & 486,0 \\
\hline 26 & 529,0 & 470,0 & $1.242,0$ & $1.082,0$ \\
\hline 52 & $1.206,0$ & 839,0 & $2.611,0$ & $2.108,0$ \\
\hline 78 & $1.750,0$ & $1.019,0$ & $4.617,0$ & $3.152,0$ \\
\hline $\mathrm{L}$ & $* * *$ & $* * *$ & $* * *$ & $* * *$ \\
\hline $\mathrm{Q}$ & ns & ns & ns & ns \\
\hline
\end{tabular}

(1) L: efeito linear de doses. ${ }^{(2)} Q$ : efeito quadrático de doses. *, $* *$ e ***: significativo $(P<0,05 ; 0,01$ e 0,001 , respectivamente). ns: não-significativo. nd: não detectado.

\section{Cobre}

Os teores de Cu extraídos do solo pelas soluções de DTPA, $\mathrm{HCl} 0,1$ mol L-1, Mehlich-1 e Mehlich-3 aumentaram significativamente de acordo com a aplicação de biossólido, com exceção doDTPA noLV, no qual as primeiras doses proporcionaram diminuição nos teores extraídos por essa solução, voltando a crescer na maior dose (Quadro 2). Essa diminuição nos teores de Cu extraídos por DTPA pode estar relacionada com o aumento do teor de matéria orgânica do solo (Figura 1), proporcionado pelo biossólido. Segundo Shuman (1988), a adição de material orgânico causa um aumentona atividade dos microrganismos, os quais adicionam agentes complexantes ao solo.

O Cu é apontado como sendo um elemento que apresenta grande afinidade pelos ligantes orgânicos do solo (McLaren \& Crawford, 1973; Mullins et al., 
Quadro 2. Teores de metais pesados extraídos dos solos com DTPA, HCl 0,1 mol L-1, Mehlich-1 e Mehlich-3

\begin{tabular}{|c|c|c|c|c|c|c|c|c|c|c|c|c|c|c|c|c|}
\hline \multirow{3}{*}{ Dose } & \multicolumn{4}{|c|}{ DTPA } & \multicolumn{4}{|c|}{$\mathrm{HCl}$} & \multicolumn{4}{|c|}{ Mehlich-1 } & \multicolumn{4}{|c|}{ Mehlich-3 } \\
\hline & \multicolumn{2}{|c|}{ LV } & \multicolumn{2}{|c|}{ RQ } & \multicolumn{2}{|c|}{ LV } & \multicolumn{2}{|c|}{$\mathbf{R Q}$} & \multicolumn{2}{|c|}{ LV } & \multicolumn{2}{|c|}{ RQ } & \multicolumn{2}{|c|}{ LV } & \multicolumn{2}{|c|}{$\mathbf{R Q}$} \\
\hline & $S C^{(1)}$ & $c c^{(2)}$ & sc & cc & sc & Cc & sc & cc & sc & cc & sc & cc & sc & cc & sc & Cc \\
\hline \multirow[t]{2}{*}{ g vaso $^{-1}$} & & & & & & & & $-\mathrm{mg} \mathrm{k}$ & $\mathrm{kg}^{-1}$ & & & & & & & \\
\hline & \multicolumn{16}{|c|}{ Cobre } \\
\hline 0 & 10,30 & 10,20 & 0,20 & 0,20 & 13,00 & 13,20 & 0,40 & 0,40 & 5,00 & 4,20 & 0,20 & 0,20 & 7,60 & 8,80 & 0,30 & 0,30 \\
\hline 13 & 7,40 & 8,50 & 0,50 & 0,40 & 13,70 & 13,70 & 0,60 & 0,70 & 5,40 & 4,40 & 0,40 & 0,40 & 8,10 & 9,10 & 0,50 & 0,50 \\
\hline 26 & 9,20 & 9,20 & 0,60 & 0,60 & 14,30 & 14,70 & 0,90 & 0,90 & 5,50 & 4,70 & 0,60 & 0,60 & 8,50 & 9,50 & 0,60 & 0,70 \\
\hline 52 & 11,80 & 5,80 & 1,00 & 1,00 & 15,30 & 15,50 & 1,80 & 1,60 & 5,80 & 5,00 & 1,10 & 1,00 & 9,10 & 9,70 & 1,00 & 0,90 \\
\hline 78 & 12,20 & 10,00 & 1,30 & 1,30 & 16,00 & 15,90 & 2,20 & 2,30 & 5,70 & 5,00 & 1,40 & 1,20 & 9,50 & 9,90 & 1,20 & 1,20 \\
\hline Média & $10,2 a^{(3)}$ & $8,7 b$ & $0,7 a$ & $0,7 a$ & $14,5 a$ & $14,6 a$ & $1,2 a$ & $1,2 a$ & $5,5 a$ & $4,7 b$ & $0,7 a$ & $0,7 a$ & $8,6 b$ & $9,4 a$ & $0,7 a$ & $0,7 a$ \\
\hline$L^{(4)}$ & $*$ & ns & $* *$ & $* *$ & $* *$ & $* *$ & $* *$ & $* *$ & $* *$ & $* *$ & $* *$ & $* *$ & $* *$ & $* *$ & $* *$ & $* *$ \\
\hline \multirow[t]{2}{*}{$\mathrm{Q}^{(5)}$} & ns & $* *$ & ns & ns & $* *$ & $* *$ & ns & ns & $* *$ & $* *$ & $*$ & ns & $* *$ & ns & ns & ns \\
\hline & & & & & & & & Manga & janês & & & & & & & \\
\hline 0 & 46,20 & 27,00 & 1,60 & 0,60 & 95,00 & 66,70 & 1,70 & 1,20 & 68,30 & 46,30 & 1,30 & 1,20 & 92,00 & 102,70 & 1,80 & 1,30 \\
\hline 13 & 53,40 & 32,60 & 1,70 & 1,40 & 111,00 & 86,00 & 2,10 & 2,00 & 83,70 & 60,70 & 1,70 & 1,50 & 128,00 & 112,30 & 2,00 & 1,80 \\
\hline 26 & 64,60 & 31,40 & 1,80 & 2,00 & 133,30 & 90,30 & 2,50 & 2,50 & 96,00 & 68,30 & 1,80 & 2,00 & 121,00 & 115,30 & 2,00 & 2,20 \\
\hline 52 & 61,00 & 66,70 & 2,60 & 3,00 & 128,30 & 144,00 & 4,00 & 4,10 & 87,70 & 111,30 & 2,50 & 3,00 & 118,00 & 137,30 & 2,70 & 2,90 \\
\hline 78 & 53,70 & 104,0 & 3,20 & 3,40 & 108,30 & 175,70 & 4,50 & 5,00 & 78,00 & 150,30 & 3,40 & 3,40 & 117,70 & 154,30 & 3,20 & 3,30 \\
\hline Média & $55,8 a$ & $52,3 a$ & $2,2 a$ & $2,1 a$ & $115,2 a$ & $112,5 a$ & $3,0 a$ & $3,0 a$ & $82,7 a$ & $87,4 a$ & $2,1 a$ & $2,2 a$ & $115,3 b$ & $124,4 a$ & $2,3 a$ & $2,3 a$ \\
\hline $\mathrm{L}$ & ns & $* *$ & $* *$ & $* *$ & ns & $* *$ & $* *$ & $* *$ & ns & $* *$ & $* *$ & $* *$ & ns & $* *$ & $* *$ & $* *$ \\
\hline $\mathrm{Q}$ & $*$ & $* *$ & $* *$ & $* *$ & $* *$ & ns & ns & ns & $* *$ & ns & ns & ns & $*$ & ns & ns & $* *$ \\
\hline & & & & & & & & Níqu & uel & & & & & & & \\
\hline 0 & 0,06 & 0,08 & 0,04 & 0,01 & 0,20 & 0,20 & 0,10 & 0,10 & 0,27 & 0,40 & 0,12 & 0,07 & 0,20 & 0,20 & 0,17 & 0,10 \\
\hline 13 & 0,10 & 0,12 & 0,09 & 0,05 & 0,23 & 0,30 & 0,23 & 0,20 & 0,40 & 0,39 & 0,20 & 0,16 & 0,20 & 0,20 & 0,27 & 0,20 \\
\hline 26 & 0,15 & 0,12 & 0,14 & 0,10 & 0,40 & 0,40 & 0,33 & 0,30 & 0,48 & 0,32 & 0,28 & 0,24 & 0,27 & 0,30 & 0,33 & 0,20 \\
\hline 52 & 0,28 & 0,21 & 0,22 & 0,19 & 0,60 & 0,63 & 0,60 & 0,50 & 0,64 & 0,45 & 0,44 & 0,43 & 0,30 & 0,37 & 0,33 & 0,30 \\
\hline 78 & 0,42 & 0,31 & 0,27 & 0,26 & 0,87 & 0,83 & 0,70 & 0,77 & 0,83 & 0,60 & 0,53 & 0,53 & 0,40 & 0,43 & 0,40 & 0,30 \\
\hline Média & $0,2 a$ & $0,16 a$ & $0,14 a$ & $0,14 a$ & $0,46 a$ & $0,46 a$ & $0,38 a$ & $0,38 a$ & $0,52 a$ & $0,44 b$ & $0,30 a$ & $0,28 b$ & $0,28 a$ & $0,3 a$ & $0,3 a$ & $0,22 b$ \\
\hline L & $* *$ & $* *$ & $* *$ & $* *$ & $* *$ & $* *$ & $* *$ & $* *$ & $* *$ & $* *$ & $* *$ & $* *$ & $* *$ & $* *$ & $* *$ & $* *$ \\
\hline Q & $* *$ & $* *$ & $* *$ & ns & ns & ns & $* *$ & ns & ns & $* *$ & $*$ & * & ns & ns & ns & $* *$ \\
\hline & & & & & & & & Chun & mbo & & & & & & & \\
\hline 0 & 0,51 & 0,57 & 0,37 & 0,27 & 0,37 & 0,40 & 0,77 & 0,63 & 0,31 & 0,41 & 0,53 & 0,56 & 0,77 & 0,77 & 0,63 & 0,70 \\
\hline 13 & 0,51 & 0,43 & 0,37 & 0,30 & 0,47 & 0,53 & 0,97 & 0,77 & 0,35 & 0,39 & 0,59 & 0,59 & 0,77 & 0,80 & 0,67 & 0,67 \\
\hline 26 & 0,52 & 0,17 & 0,37 & 0,35 & 0,60 & 0,73 & 1,07 & 0,90 & 0,39 & 0,24 & 0,63 & 0,63 & 0,80 & 0,73 & 0,77 & 0,70 \\
\hline 52 & 0,65 & 0,21 & 0,44 & 0,40 & 0,67 & 0,93 & 1,27 & 1,23 & 0,44 & 0,36 & 0,76 & 0,79 & 0,87 & 0,83 & 1,00 & 0,80 \\
\hline 78 & 0,75 & 0,27 & 0,42 & 0,41 & 0,80 & 1,13 & 1,27 & 1,43 & 0,48 & 0,41 & 0,84 & 0,84 & 1,03 & 0,97 & 0,90 & 0,97 \\
\hline Média & $0,60 a$ & $0,36 b$ & $0,40 a$ & $0,36 \mathrm{~b}$ & $0,60 \mathrm{~b}$ & $0,72 a$ & 1,10a & $0,98 a$ & $0,38 a$ & $0,36 \mathrm{~b}$ & $0,66 a$ & $0,68 a$ & $0,86 a$ & $0,82 a$ & $0,80 a$ & $0,78 a$ \\
\hline L & $* *$ & $* *$ & $* *$ & $* *$ & $* *$ & $* *$ & $* *$ & $* *$ & $* *$ & $\mathrm{~ns}$ & $* *$ & $* *$ & $* *$ & $* *$ & $* *$ & $* *$ \\
\hline Q & $* *$ & $* *$ & ns & ns & ns & ns & $* *$ & ns & ns & $* *$ & ns & ns & ns & $* *$ & ns & ns \\
\hline & & & & & & & & Zin & ICo & & & & & & & \\
\hline 0 & 0,70 & 0,80 & 0,40 & 0,30 & 1,60 & 1,50 & 0,60 & 0,60 & 1,00 & 1,10 & 0,40 & 0,40 & 0,70 & 0,80 & 0,60 & 0,60 \\
\hline 13 & 1,70 & 2,10 & 3,30 & 2,70 & 5,10 & 4,90 & 4,10 & 4,10 & 3,90 & 3,60 & 3,20 & 3,30 & 2,20 & 2,30 & 3,10 & 3,00 \\
\hline 26 & 3,60 & 4,00 & 5,00 & 5,30 & 9,20 & 9,00 & 7,40 & 6,90 & 7,10 & 7,20 & 7,00 & 6,70 & 4,20 & 4,20 & 5,60 & 5,50 \\
\hline 52 & 9,50 & 4,70 & 9,40 & 10,60 & 16,70 & 17,50 & 15,30 & 15,90 & 12,50 & 11,30 & 12,80 & 11,80 & 8,60 & 8,50 & 10,80 & 10,70 \\
\hline 78 & 14,10 & 11,90 & 14,70 & 15,00 & 23,70 & 24,00 & 21,00 & 24,30 & 19,20 & 18,00 & 17,30 & 18,10 & 13,50 & 13,10 & 15,60 & 15,40 \\
\hline Média & $5,92 a$ & $4,70 b$ & $6,56 a$ & $6,78 a$ & $11,26 a$ & $11,38 a$ & $9,68 b$ & $10,36 a$ & $8,74 a$ & $8,24 a$ & $8,14 a$ & $8,06 a$ & $5,84 a$ & $5,78 a$ & $7,14 a$ & $7,04 a$ \\
\hline L & $* *$ & $* *$ & $* *$ & $* *$ & $* *$ & $* *$ & $* *$ & $* *$ & $* *$ & $* *$ & $* *$ & $* *$ & $* *$ & $* *$ & ** & $* *$ \\
\hline $\mathrm{Q}$ & $*$ & $*$ & ns & ns & ns & ns & ns & * & * & ns & ns & ns & $* *$ & $* *$ & ns & ns \\
\hline
\end{tabular}

(1) sc: tratamentos sem aplicação de corretivos. ${ }^{(2)}$ cc: tratamentos com aplicação de corretivos. ${ }^{(3)}$ médias na mesma linha, para o mesmo solo, seguidas de letras iguais não diferem entre si pelo teste $F(P<0,05) .{ }^{(4)} \mathrm{L}$ : efeito linear de doses. ${ }^{(5)} \mathrm{Q}$ : efeito quadrático de doses. * e **: significativo ( $P<0,05$ e 0,01, respectivamente). ns: não-significativo. 
1982). Desse modo, apenas o complexante DTPA pode ter sido sensível a essa afinidade do $\mathrm{Cu}$ pelos ligantes orgânicos. Deve-se observar também que, nas menores doses de bi ossólido, as quantidades de $\mathrm{Cu}$ aplicadas foram pequenas comparadas àquelas das maiores doses. O mesmo comportamento não foi observado no RQ, provavelmente pelos baixos teores naturais do nutriente nesse sol o.

A aplicação de corretivos causou efeito significativo na extração de $\mathrm{Cu}$ apenas para as soluções de DTPA, Mehlich-1 e Mehlich-3, para o LV (Quadro 4), no qual ocorreram as maiores variações do $\mathrm{pH}$ entre os tratamentos com e sem corretivos, em relação ao RQ (Figura 2). Deve-se observar, entretanto, que os teores de Cu determinados pelo Mehlich-3 aumentaram com aplicação de corretivos, comportamento contrário ao observado para Mehlich-1 eDTPA. Camargo et al. (1982) já haviam encontrado correlação negativa entre o pH do solo e o teor de Cu extraído com DTPA, indicando que esse extrator foi sensível à mudança no $\mathrm{pH}$ do solo.

O Cu acumulado na parte aérea das plantas relacionou-se significativamente com os teores do elemento extraídos pelas quatro soluções, para ambos os solos, exceção feita ao DTPA no LV (Quadro 3). O HCl destacou-se por apresentar os maiores valores de coeficientes de determinação
(Quadro 3), considerando-se os dois solos. Os resultados encontrados na literatura, com relação à eficiência dos extratores para determinação da disponibilidade deCu para plantas, são controversos. Haq \& Miller (1972) também relataram que oDTPA não foi eficiente na avaliação da disponibilidade de $\mathrm{Cu}$ para plantas de milho e beterraba, respectivamente. Entretanto, Sanders et al. (1986) e Berton et al. (1997) encontraram coeficientes de correlação significativos entre o Cu na planta e o extraído do solo por DTPA, após aplicação de biossólido. Mulchi et al. (1991) também, após aplicação de biossólido no solo, verificaram que o Cu extraído pelas soluções DTPA, Mehlich-1 e Mehlich-3 foi correlacionado com a concentração de Cu na parte aérea de milho.

O ajuste de regressão múltipla das quantidades de cobre acumulado na parte aérea do milho, como variável dependente dos teores do metal extraído do solo pelas soluções e os valores de $\mathrm{pH}$, indicou que a inclusão do $\mathrm{pH}$ foi significativa apenas para os teores determinados por Mehlich-1 e Mehlich-3, no LV (Quadro 3), resultado semel hante ao de Sanders et al. (1986) que não verificaram aumento nos coeficientes de determinação das equações lineares obtidas do teor de Cu na planta e no solo determinado pelo DTPA, quando foram incluídos no model o os valores de $\mathrm{pH}$ do solo.

Quadro 3. Equações de regressão múltipla e coeficientes de determinação das quantidades de cobre acumulado na parte aérea do $\mathrm{mi}^{\mathrm{I} h o}\left(\mu \mathrm{g} \mathrm{vaso}^{-1}\right)$ como variável dependente dos teores do metal extraído do solo $\left(\mathrm{mg} \mathrm{kg}^{-1}\right)$ pelas soluções e os valores de $\mathrm{pH}$

\begin{tabular}{|c|c|c|}
\hline Extrator & E quação de regressão & $\mathbf{R}^{2}$ \\
\hline \multicolumn{3}{|c|}{ Cobre acumulado na parte aérea $(\hat{y})$} \\
\hline \multicolumn{3}{|c|}{ LV } \\
\hline DTPA & $\hat{y}=12,1+4,67 \mathrm{DTPA}$ & $0,116^{\text {ns }}$ \\
\hline $\mathrm{HCl}$ & $\hat{y}=-273,0+22,7 \mathrm{HCl}$ & $0,834 * * *$ \\
\hline \multirow[t]{2}{*}{ Mehlich-1 (M 1) } & $\hat{y}=-58,3+22,6 \mathrm{M} 1$ & $0,190 *$ \\
\hline & $\hat{y}=-530,3+63,8 \mathrm{M} 1+55,8 \mathrm{pH}$ & $0,777^{* * *}$ \\
\hline \multirow[t]{3}{*}{ Mehlich-3 (M 3) } & $\hat{y}=-207,8+29,4 \mathrm{M} 3$ & $0,604^{* * *}$ \\
\hline & $\hat{y}=-207,8+46,57 M 3-32,66 \mathrm{pH}$ & $0,816^{* * *}$ \\
\hline & \multicolumn{2}{|l|}{$\mathrm{RQ}$} \\
\hline DTPA & $\hat{y}=0,130+67,913 D T P A$ & $0,935^{* * *}$ \\
\hline $\mathrm{HCl}$ & $\hat{y}=5,51+35,61 \mathrm{HCl}$ & $0,880 * * *$ \\
\hline Mehlich-1 (M1) & $\hat{y}=3,79+63,46 \mathrm{M} 1$ & $0,943^{* * *}$ \\
\hline Mehlich-3 (M3) & $\hat{y}=-12,7+84,1 \mathrm{M} 3$ & $0,936 * * *$ \\
\hline
\end{tabular}

* e***: significativo ( $P<0,05$ e 0,001, respectivamente). ns: não-significativo. 


\section{Manganês}

Os teores de Mn extraídos do solo pelas quatro soluções aumentaram linearmente de acordo com as doses de biossólido, com exceção dos tratamentos que não receberam calagem no LV (Quadro 2). Nesses tratamentos, ocorreram respostas quadráticas dos teores de $\mathrm{Mn}$, com redução nas duas maiores doses. Na figura 2, observa-se que os valores de $\mathrm{pH}$ nessas doses foram mais elevados, o que poderia justificar a menor extração. Entretanto, esse não deve ter sido o único mecanismo envolvido na redução da disponibilidade, uma vez que, nos tratamentos com calagem (nas mesmas doses), nos quais o pH foi superior, o mesmo não ocorreu.

Relacionando as quantidades de $\mathrm{Mn}$ aplicado pelo biossólido e as do $M n$ extraído pelas soluções, verificou-se que, noRQ, foram recuperados, em média, $42,64,41$ e $33 \%$ do $\mathrm{Mn}$, pel as soluções DTPA, $\mathrm{HCl}$, Mehlich-1 e Mehlich-3, respectivamente. No LV, foram "recuperados" 649, 941, 842 e $493 \%$ do Mn, pelas mesmas soluções. I sso pode indicar que, para o solo LV , a aplicação de biossól ido promoveu maior solubilização do manganês do solo. Este resultado foi confirmado pelo estudo de fracionamento do metal (Borges \& Coutinho, 2004). Shuman (1988) verificou a ocorrência de uma redistribuição do Mn do solo de formas menos disponíveis para formas mais disponíveis após adicionar restos da cultura detrigo para aumentar os teores de matéria orgânica de oito solos. O autor atribuiu essa redistribuição à redução do potencial redox do solo provocada pela decomposição da matéria orgânica. A ausência do mesmo efeito no RQ pode ser explicada pelos baixos teores nativos do el emento nesse solo.

Observou-se que a reação do solo afetou marcadamente as quantidades de $\mathrm{Mn}$ absorvido pelas plantas, no LV (Quadro 1). Entretanto, verificou-se que o efeito do $\mathrm{pH}$ do solo sobre a solubilidade de $\mathrm{Mn}$ nos extratores não ficou claro, em razão, possivelmente, das diferentes condições de oxirredução. Abreu et al. (1994b) nãoverificaram diminuição na extração de $\mathrm{Mn}$ com o aumento do $\mathrm{pH}$ do solo, proporcionado pela aplicação de doses de corretivos, utilizando os extratores DTPA, $\mathrm{HCl}$ e Mehlich-1. Por outro lado, Camargo et al. (1982) encontraram alta correlação entre os diferentes valores de $\mathrm{pH}$ do solo e os teores de $\mathrm{Mn}$ extraídos pelo DTPA, mostrando que esse extrator consegue discriminar o comportamento do micronutriente a diferentes valores de $\mathrm{pH}$.

F oram obtidos coeficientes de determinação significativos, embora baixos, entre as quantidades acumuladas de $\mathrm{Mn}$ na parte aérea das plantas e o manganês extraído do LV pelas quatro soluções (Quadro 4). Para oRQ, os coeficientes de determinação foram altamente significativos e mais elevados. Abreu et al. (1994a) encontraram coeficientes de correl ação entreo Mn acumulado na parte aérea da soja eo extraído em 10 sol os da ordem de 0,836**, 0,844** e 0,841**, para oDTPA, Mehlich-1 e HCl, respectivamente.
A inclusão da variável pH melhorou significativamente a relação entre o manganês acumulado na planta e o determinado por meio dos extratores, no LV, permitindo uma melhor interpretação da disponi bilidade do metal para as plantas (Quadro 4). Esses resultados vão ao encontro dos observados por Abreu et al. (1994a) que relataram que os extratores DTPA, Mehlich-1 e HCl só foram eficazes em avaliar o $\mathrm{Mn}$ disponível para plantas de soja quando a interpretação foi acompanhada do valor de pH. No $R Q$, houve efeito significativo da inclusão da variável $\mathrm{pH}$ apenas para os extratores DTPA e Mehlich-3, graças provavel mente, à menor variação nos val ores de $\mathrm{pH}$ ocorrida nesse solo, em comparação ao LV (Figura 2).

\section{Níquel}

Observaram-se aumentos lineares dos teores de $\mathrm{Ni}$ determinados pelos extratores de acordo com a adição de biossólido (Quadro 4).

Os teores de Ni no solo, mesmo após aplicação do biossólido, podem ser considerados normais comparados com aqueles relatados por Abreu et al. (1995), queencontraram uma variação de 0,09 a 0,90; 0,13 a 0,62 e 0,41 a 1,90 $\mathrm{mg} \mathrm{kg}^{-1}$, para DTPA, Mehlich-1 eM ehlich-3, respectivamente, em 31 sol os não contami nados do estado deSão Paulo. Os teores do metal encontrados no presente trabal hoestiveram abaixo daquele apontado como tóxico por Dang et al . (1990) para a cultura da cebola (29 $\mathrm{g} \mathrm{kg}^{-1}$ pelo DTPA).

As análises de regressão (Quadro 5) mostraram que os teores de $\mathrm{Ni}$ no solo, determinados pelos quatro extratores, estiveram relacionados com a quantidade acumulada do metal na parte aérea das plantas, no RQ. Entretanto, o M ehlich-3 apresentou o menor valor de $R^{2}\left(0,185^{*}\right)$. No LV, as quantidades acumuladas de $\mathrm{Ni}$ na planta relacionaram-se significativamente com os teores desse el emento no solo determinado apenas pelo DTPA; entretanto, seu valor foi baixo, mostrando que essas não foram eficientes em determinar a disponibilidade do $\mathrm{Ni}$ nesse solo.

A aplicação de corretivos afetou signi ficativamente os teores de $\mathrm{Ni}$ extraídos por Mehlich-1, em ambos os solos, e Mehlich-3, no RQ (Quadro 4). Todavia, o efeito da aplicação de corretivos não foi claro.

O ajuste de regressão múltipla das quantidades de $\mathrm{Ni}$ acumulado na parte aérea do milho, como variável dos teores do metal extraído do solo pelas soluções e os val ores de pH, indicou que, para o LV, apenas para o DTPA essas variáveis explicaram, de forma significativa, o $\mathrm{Ni}$ acumulado, embora o coeficiente de determinação tenha sido baixo (Quadro 5). NoRQ, a inclusão dopH foi significativa, quando foram utilizadas as soluções DTPA e Mehlich-3. Abreu et al. (1995), comparando os métodos de Mehlich-1, Mehlich-3 e DTPA, verificaram, também, que a inclusão de outras propriedades do solo, como val or de $\mathrm{pH}$ para o $\mathrm{Ni}$, e 
Quadro 4. Equações de regressão múltipla e coeficientes de determi nação das quantidades de manganês acumulado na parte aérea do $\mathrm{mi}^{\mathrm{l}} \mathrm{ho}\left(\mu \mathrm{g} \mathrm{vaso}^{-1}\right)$ como variável dependente dos teores do metal extraído do solo $\left(\mathrm{mg} \mathrm{kg}^{-1}\right)$ pelas soluções e os valores de $\mathrm{pH}$

\begin{tabular}{|c|c|c|}
\hline Extrator & E quação de regressão & $\mathbf{R}^{2}$ \\
\hline \multicolumn{3}{|c|}{ Manganês acumulado na parte aérea ( $\hat{y}$ ) } \\
\hline \multicolumn{3}{|c|}{ LV } \\
\hline \multirow[t]{2}{*}{ DTPA } & $\hat{y}=569,3+71,2$ DTPA & $0,347^{* * *}$ \\
\hline & $\hat{y}=16894,3+67,5$ DTPA $-3423,8 p H$ & $0,754 * * *$ \\
\hline \multirow[t]{2}{*}{$\mathrm{HCl}$} & $\hat{y}=-2430,3+60,2 \mathrm{HCl}$ & $0,439 * * *$ \\
\hline & $\hat{y}=14126,2+58,7 \mathrm{HCl}-3479,1 \mathrm{pH}$ & $0,859 * * *$ \\
\hline \multirow[t]{2}{*}{ Mehlich-1 (M 1) } & $\hat{y}=-123,3+53,4 M 1$ & $0,300 * *$ \\
\hline & $\hat{y}=17611,3+60,3 \mathrm{M} 1-3889,8 \mathrm{pH}$ & $0,821^{* * *}$ \\
\hline \multirow[t]{2}{*}{ Mehlich-3 (M 3) } & $\hat{y}=-1954,8+53,2 M 3$ & $0,111^{*}$ \\
\hline & $\hat{y}=14437,8+91,2 \mathrm{M} 3-4449,1 \mathrm{pH}$ & $0,742 * *$ \\
\hline \multicolumn{3}{|c|}{$\mathrm{RQ}$} \\
\hline \multirow[t]{2}{*}{ DTPA } & $\hat{y}=-449,4+728,4$ DTPA & $0,838 * * *$ \\
\hline & $\hat{y}=-2452,2+693,8 D T P A+437,5 p H$ & $0,892 * * *$ \\
\hline $\mathrm{HCl}$ & $\hat{y}=-274,4+467,8 \mathrm{HCl}$ & $0,764 * * *$ \\
\hline Mehlich-1 (M 1) & $\hat{y}=-546,3+758,7 M 1$ & $0,802 * * *$ \\
\hline \multirow[t]{2}{*}{ Mehlich-3 (M 3) } & $\hat{y}=-1093,4+946,5 \mathrm{M} 3$ & $0,829 * * *$ \\
\hline & $\hat{y}=-2673,2+899,2 \mathrm{M} 3+356,1 \mathrm{pH}$ & $0,864 * * *$ \\
\hline
\end{tabular}

*,** e ***: significativo ( $\mathrm{P}<0,05 ; 0,01$ e 0,001, respectivamente). ns: não-significativo.

o teor do elemento no solo, como variáveis independentes no model o de regressão, aumentaram os coeficientes de determinação.

\section{Chumbo}

As quantidades de $\mathrm{Pb}$ extraídas dos solos aumentaram de acordo com as doses de biossólido, para todos os extratores, com exceção do DTPA e Mehlich-1 no LV, na presença de corretivos, para o qual os teores diminuíram nas primeiras doses, voltando a crescer (Quadro 4). Esse fato pode estar relacionado com o aumento do teor de matéria orgânica do solo (Figura 1), causado pela aplicação de biossólido. Da mesma forma, MacLean (1969) relatou diminuição na absorção de $\mathrm{Pb}$ por plantas com o aumento do teor de matéria orgânica e pH. De acordo com esse autor, o íon $\mathrm{Pb}^{2+}$ pode formar complexos com compostos húmi cos do sol o e o efeito da calagem foi, provavelmente, associado à maior capacidade da matéria orgânica em complexar o metal com o aumento do pH do solo. Após aplicar várias fontes de material orgânico ao solo, Shuman (1998) verificou, por meio da técnica de fracionamento, que a maior parte do $\mathrm{Pb}$ adicionado esteve associado à fração orgânica. Deve-se observar que as quantidades do metal adicionadas for am pequenas.

Comparando os resultados obtidos com os extratores Mehlich-1 e DTPA, observou-se que a solução ácida (Mehlich-1) dissolveu maiores quantidades de $\mathrm{Pb}$ que o complexante (DTPA) no RQ. Entretanto, no LV, ocorreu o inverso, tendo o DTPA extraído mais do metal do que o Mehlich-1. Resultados semel hantes foram encontrados por Li \& Shuman (1997), ao empregarem DTPA e Mehlich-1 para determinar os teores de $\mathrm{Pb}$. Esses autores encontraram, ainda, coeficientes de correl ação negativos e significativos entre o Pb extraído pelo Mehlich-1 e o conteúdo de argila e de óxidos de Fe e Al dos solos, mostrando que esses materiais desempenham importante papel na adsorção e readsorção do metal no sol o durante o procedimento de extração. O mesmo não foi verificado para o DTPA.

A mesma observação pode ser feita na comparação entre o HCl e o Mehlich-3. A maior extração de $\mathrm{Pb}$ pelo Mehlich-3 no LV em relação ao $\mathrm{HCl}$ deveu-se, provavelmente, à presença de um agente 


\section{Quadro 5. Equações de regressão múltipla e coeficientes de determinação das quantidades de níquel acumulado na parte aérea do $\mathrm{mi}^{\mathrm{l} h o}\left(\mu \mathrm{g} \mathrm{vaso}^{-1}\right)$ como variável dependente dos teores do metal extraído do solo $\left(\mathrm{mg} \mathrm{kg}^{-1}\right)$ pelas soluções e os valores de $\mathrm{pH}$}

\begin{tabular}{|c|c|c|}
\hline Extrator & Equação de regressão & $\mathbf{R}^{2}$ \\
\hline \multicolumn{3}{|c|}{ Níquel acumulado na parte aérea $(\hat{y})$} \\
\hline DTPA & $\begin{array}{c}\text { LV } \\
\hat{y}=5,95+12,2 \text { DTPA } \\
\hat{y}=-7,71+11,84 \text { DTPA }+2,91 p H\end{array}$ & $\begin{array}{l}0,124^{*} \\
0,278^{*}\end{array}$ \\
\hline $\mathrm{HCl}$ & $\hat{y}=5,77+5,21 \mathrm{HCl}$ & $0,104^{\text {ns }}$ \\
\hline Mehlich-1 (M1) & $\hat{\mathrm{y}}=5,84^{*}+4,95 \mathrm{M} 1$ & $0,043^{n s}$ \\
\hline Mehlich-3 (M3) & $\hat{y}=5,17+10,6 \mathrm{M} 3$ & $0,058^{\text {ns }}$ \\
\hline \multicolumn{3}{|c|}{$\mathrm{RQ}$} \\
\hline DTPA & $\begin{array}{l}\hat{y}=0,150+80,2 \text { DTPA } \\
\hat{y}=-34,037+75,716 \text { DTPA }+7,333 \mathrm{pH}\end{array}$ & $\begin{array}{l}0,580^{* * *} \\
0,659^{* * *}\end{array}$ \\
\hline $\mathrm{HCl}$ & $\hat{y}=-1,32+32,70 \mathrm{HCl}$ & $0,641^{* * *}$ \\
\hline Mehlich-1 (M 1) & $\hat{y}=-2,08+44,17 M 1$ & $0,574^{* * *}$ \\
\hline Mehlich-3 (M3) & $\begin{array}{l}\hat{y}=0,492+44,8 \mathrm{M} 3 \\
\hat{y}=-58,3+51,134 \mathrm{M} 3+11,834 \mathrm{pH}\end{array}$ & $\begin{array}{l}0,185^{*} \\
0,390^{* * *}\end{array}$ \\
\hline
\end{tabular}

*,** e***: significativo ( $P<0,05 ; 0,01$ e 0,001, respectivamente). ns: não-significativo.

complexante (EDTA) na sua composição. No solo arenoso(RQ), o $\mathrm{HCl}$ extraiu as mai ores quantidades do metal.

A aplicação de corretivos causou efeito significativo na extração do $\mathrm{Pb}$ apenas para o DTPA, em ambos os solos (Quadro 4). O Pb extraído pelas soluções Mehlich-1 e HCl foi influenciadosignificativamente pela aplicação de corretivos apenas no LV. Li \& Shuman (1997) encontraram coeficiente de correlação significativo e negativo entre o pH dos solos e os teores de $\mathrm{Pb}$ por Mehlich-1, o mesmo não acontecendo para o DTPA.

Os teores máximos de $\mathrm{Pb}$ no solo foram de 0,82 ; 1,47; 0,84 e 1,10 $\mathrm{mg} \mathrm{kg}^{-1}$ extraídos pelo DTPA, $\mathrm{HCl}$, Mehlich-1 e Mehlich-3, respectivamente. Esses val ores estão dentro da faixa encontrada por Abreu et al. (1995) para 31 sol os não contaminados do estado de São Paulo (0,6-5,9; 1,1-2,6 e 4,6-11,0 $\mathrm{mg} \mathrm{kg}^{-1}$ para DTPA, Mehlich-1 e Mehlich-3, respectivamente) e se encontram abaixo de $20 \mathrm{mg} \mathrm{kg}^{-1}$, extraído com DTPA, considerado "alto" por Wallace \& Wallace (1994).

Verificou-se que apenas os extratores DTPA e $\mathrm{HCl}$ apresentaram relação significativa para o $\mathrm{Pb}$ acumulado na parte aérea das plantas cultivadas no LV (Quadro 6). No RQ, apenas o Mehlich-3 apresentou relação significativa, entretanto o valor de $\mathrm{R}^{2}$ obtido foi baixo. A inclusão dos valores de $\mathrm{pH}$ como variável independenteno model o de regressão, para aumentar a capacidade de predição do acúmulo de $\mathrm{Pb}$ na parte aérea do milho, foi significativa somente para os mesmos casos (Quadro 6). Os extratores DTPA, HCl Mehlich-1 e Mehlich-3 vêm sendo testados sem sucesso para a avaliação da disponibilidade de $\mathrm{Pb}$ para várias culturas (Mulchi et al., 1991; Abreu et al., 1995).

\section{Zinco}

Os teores de Zn do solo, extraídos pelas quatro soluções testadas, aumentaram linearmente de acordo com as doses de biossól ido (Quadro 4).

As soluções de $\mathrm{HCl}$ e Mehlich-1 recuperaram praticamenteas mesmas proporções de Zn em ambos os solos (29,0 e $22,0 \%$, respectivamente). Os extratores DTPA e M ehlich-3 recuperaram mais do metal aplicado no RQ (18,5 e19,3\%, respectivamente), em relação ao LV (15,5 e 16,3 \%, respectivamente). A maior variação na recuperação do Zn entre os dois solos, verificada para esses extratores, pode indicar maior sensi bilidade dessas sol uções aos atributos do solo relacionados com o fator capacidade do nutriente no solo, em relação ao HCl e Mehlich-1. O mesmo resultado foi encontrado por Oliveira et al. (1999) que estudaram a disponibilidade de Zn em 12 solos, 


\section{Quadro 6. Equações de regressão múlti pla e coeficientes de determi nação das quantidades de chumbo acumulado na parte aérea do $\mathrm{mi}^{\mathrm{l}} \mathrm{ho}\left(\mu \mathrm{g} \mathrm{vaso}^{-1}\right)$ como variável dependente dos teores do metal extraído do solo $\left(\mathrm{mg} \mathrm{kg}^{-1}\right)$ pelas soluções e valores de $\mathrm{pH}$}

\begin{tabular}{|c|c|c|}
\hline Extrator & E quação de regressão & $\mathbf{R}^{2}$ \\
\hline \multicolumn{3}{|c|}{ Chumbo acumulado na parte aérea ( $\hat{y}$ ) } \\
\hline \multicolumn{3}{|c|}{ LV } \\
\hline \multirow[t]{2}{*}{ DTPA } & $\hat{y}=54,15-84,21 \mathrm{DTPA}$ & $0,633^{* * *}$ \\
\hline & $\hat{y}=-5,37-68,3 D T P A+11,0 p H$ & $0,684^{* * *}$ \\
\hline \multirow[t]{2}{*}{$\mathrm{HCl}$} & $\hat{y}=-33,0+69,9 \mathrm{HCl}$ & $0,623 * * *$ \\
\hline & $\hat{y}=-90,98+56,34 \mathrm{HCl}+14,21 \mathrm{pH}$ & $0,723^{* *}$ \\
\hline Mehlich-1 (M 1) & $\hat{y}=37,8-64,7 M 1$ & $0,048^{\text {ns }}$ \\
\hline Mehlich-3 (M 3) & $\hat{y}=-17,1+36,5 \mathrm{M} 3$ & $0,039^{\text {ns }}$ \\
\hline \multicolumn{3}{|c|}{$\mathrm{RQ}$} \\
\hline DTPA & $\hat{y}=2,35-3,56 \mathrm{DTPA}$ & $0,008^{\text {ns }}$ \\
\hline $\mathrm{HCl}$ & $\hat{y}=3,45-2,34 \mathrm{HCl}$ & $0,074^{\text {ns }}$ \\
\hline Mehlich-1 (M1) & $\hat{y}=4,98-5,85 \mathrm{M} 1$ & $0,091^{\text {ns }}$ \\
\hline \multirow[t]{2}{*}{ Mehlich-3 (M 3) } & $\hat{\mathrm{y}}=5,37-5,57 \mathrm{M} 3$ & $0,136 *$ \\
\hline & $\hat{\mathrm{y}}=15,9-4,42 \mathrm{M} 3-2,40 \mathrm{pH}$ & $0,284 *$ \\
\hline
\end{tabular}

*,** e***: significativo ( $\mathrm{P}<0,05 ; 0,01$ e 0,001, respectivamente). ns: não-significativo.

utilizando as mesmas sol uções do presentetrabalho. De acordo com esses autores, a menor sensibilidade do $\mathrm{HCl}$ e Mehlich-1 se deve ao maior poder de extração dessas soluções e conseqüente menor desgaste delas em condições mais tamponadas.

As análises de regressão entre os teores de $Z n$ extraídos do solo pelos extratores e o teor e quantidade do nutriente na parte aérea das plantas mostraram que todas as soluções foram eficientes na avaliação da disponibilidade do nutriente para as plantas (Quadro 7). A eficiência desses extratores para avaliação de Zn para plantas foi comprovada por vários autores (Ritchey et al., 1986; Bataglia \& Raij, 1989; Mulchi et al., 1991).

Verifica-se, na literatura, correlação significativa e negativa entre o pH do solo e a absorção de $\mathrm{Zn}$ pelas plantas (Haq \& Miller, 1972; Camargo et al., 1982; Oliveira et al., 1999). No RQ, a aplicação de corretivos causou efeito significativo na extração de zinco apenas pel o extrator $\mathrm{HCl}$ (Quadro 4). Deve-se, no entanto, observar que esse extrator não refletiu adequadamente a dimi nuição da disponibilidade do Zn para as plantas após a calagem.

No LV, no qual a variação de $\mathrm{pH}$ entre os tratamentos com e sem corretivos foi maior
(Figura 2), verificou-se efeito significativo da aplicação de corretivos na extração do el emento pelo DTPA (Quadro 4). A mai or sensibilidade do DTPA a alterações na disponibilidade de Zn, decorrente da variações no $\mathrm{pH}$ do solo, foi apontada por diversos autores (Korcak \& Fanning, 1978; Camargo et al., 1982; Shuman, 1986).

As equações de regressão múlti pla, determinadas entre o Zn acumulado na parte aérea do milho, dependendo do $\mathrm{pH}$ e dos teores do elemento no solo, estão apresentadas no quadro 7. Verificou-se, pelos resultados, que a inclusão do $\mathrm{pH}$ como variável independente proporcionou aumento (embora pequeno) da capacidade de predição do acúmulo de Zn na parte aérea pelas soluções em ambos os solos, com exceção do DTPA no LV. Esse fato se deve, provavel mente, à maior sensi bilidade desse extrator ao efeito do $\mathrm{pH}$ sobre a disponibilidade do nutriente, como já foi discutido anteriormente.

\section{CONCLUSÕES}

1. Os extratores não se mostraram eficientes na avaliação conjunta de todos os metais. 
Quadro 7. Equações de regressão múltipla e coeficientes de determinação das quantidades de zinco acumulado na parte aérea do $\mathrm{mi}^{\mathrm{l} h o}\left(\mu \mathrm{g} \mathrm{vaso}^{-1}\right)$ como variável dependente dos teores do metal extraído do solo $\left(\mathrm{mg} \mathrm{kg}^{-1}\right)$ pelas soluções e valores de pH

\begin{tabular}{|c|c|c|}
\hline Extrator & E quação de regressão & $\mathbf{R}^{2}$ \\
\hline \multicolumn{3}{|c|}{ Zinco acumulado na parte aérea $(\hat{y})$} \\
\hline \multicolumn{3}{|c|}{ LV } \\
\hline DTPA & $\hat{y}=94,0+105,7 \mathrm{DTPA}$ & $0,867 * * *$ \\
\hline \multirow[t]{2}{*}{$\mathrm{HCl}$} & $\hat{y}=-2,94+58,1 \mathrm{HCl}$ & $0,827 * * *$ \\
\hline & $\hat{y}=791,93+59,85 \mathrm{HCl}-172,86 \mathrm{pH}$ & $0,857 * * *$ \\
\hline \multirow[t]{2}{*}{ Mehlich-1 (M 1) } & $\hat{y}=-\quad 4,93+77,8 M_{1}$ & $0,867 * * *$ \\
\hline & $\hat{y}=660,21+79,26 \mathrm{M} 1-143,85 \mathrm{pH}$ & $0,887 * * *$ \\
\hline \multirow[t]{2}{*}{ Mehlich-3 (M 3) } & $\hat{y}=44,3+105,2 \mathrm{M} 3$ & $0,847 * * *$ \\
\hline & $\hat{y}=836,0+108,1 \mathrm{M} 3-171,8 \mathrm{pH}$ & $0,876 * * *$ \\
\hline \multicolumn{3}{|c|}{$\mathrm{RQ}$} \\
\hline \multirow[t]{2}{*}{ DTPA } & $\hat{y}=-132,2+261,3 D T P A$ & $0,911 * * *$ \\
\hline & $\hat{y}=2775,4+274,9 D T P A-613,6 p H$ & $0,935^{* * *}$ \\
\hline \multirow[t]{2}{*}{$\mathrm{HCl}$} & $\hat{y}=11,3+162,1 \mathrm{HCl}$ & $0,841 * * *$ \\
\hline & $\hat{y}=3226,3+172,7 \mathrm{HCl}-704,5 \mathrm{pH}$ & $0,871 * * *$ \\
\hline \multirow[t]{2}{*}{ Mehlich-1 (M 1) } & $\hat{y}=-141,1+216,2 M 1$ & $0,893 * * *$ \\
\hline & $\hat{y}=2618,5+226,7 M 1-599,5 p H$ & $0,915^{* * *}$ \\
\hline \multirow[t]{2}{*}{ M ehlich-3 (M 3) } & $\hat{y}=-188,8+253,8 \mathrm{M} 3$ & $0,918 * * *$ \\
\hline & $\hat{\mathrm{y}}=2501,1+265,4 \mathrm{M} 3-584,1 \mathrm{pH}$ & $0,938 * * *$ \\
\hline
\end{tabular}

***: significativo $(\mathrm{P}<0,01)$.

2. A inclusão do pH do solo nos modelos de regressão melhorou significativamente a relação entreo Mn na planta eoteor de Mn pelos extratores.

3. Para o solo argiloso, a inclusão do pH mel horou significativamente a relação entre o Cu na planta e o teor deCu pel os extratores, com exceção do DTPA.

\section{LITE RATURA CITADA}

ABREU, C.A.; ABREU, M.F.; RAIJ , B.van. \& SANTOS, W.R. Comparação de métodos de análise para avaliar a disponibilidade de metais pesados em solos. R. Bras. Ci. Solo, 19:463-468, 1995.

ABREU, C.A.; NOVAIS, R.F.; RAIJ , B.van. \& RIBEIRO, A.C. Comparação de métodos de análise para avaliar a disponibilidade do manganês em solos. R. Bras. Ci. Solo, 18:81-90, 1994a.

ABREU, C.A.; NOVAIS, R.F.; RAIJ , B.van. \& RIBEIRO, A.C. Influência da reação do solo na extração de manganês por diferentes extratores químicos. R. Bras. Ci. Solo, 18:91-99, 1994b.
BATAGLIA, O.C. \& RAIJ , B. van. Eficiência de extratores de micronutrientes na análise de solo. R. Bras. Ci. Solo, 13:205212, 1989.

BATAGLIA, O.C.; FURLANI, A.M.C.; TEIXEIRA, J.P.F.; FURLANI, P.R. \& GALLO, J.R. Métodos de análise química de plantas. Campinas, Instituto Agronômico de Campinas, 1983. 48p. (Boletim Técnico, 78)

BERTON, R.S.; CAMARGO, O.A. \& VALADARES, J.M.A.S. Absorção de nutrientes pel o mil ho em resposta à adição de lodo de esgoto a cinco solos paulistas. R. Bras. Ci. Solo, 13:187-192, 1989.

BERTON, R.S.; VALADARES, J.M.A.S.; CAMARGO, O.A. \& BATAGLIA, O.C. Pel etização do lodo de esgoto e adição de $\mathrm{CaCO}_{3}$ na produção de matéria seca e absorção de $\mathrm{Zn}$, Cu e $\mathrm{Ni}$ pelo milho em três Latossolos. R. Bras. Ci. Solo, 21:685691, 1997.

BORGES, M.R. \& COUTINHO, E.L.M. Metais pesados do solo após aplicação de biossólido. I - Fracionamento. R. Bras. Ci. Solo, 28:543-555, 2004

CAMARGO, O.A.; VALADARES, J.M.A.S. \& DECHEN, A.R. Efeitos do $\mathrm{pH}$ e da incubação na extração do manganês, zinco, cobre e de ferro do solo. R. Bras. Ci. Solo, 6:83-88, 1982. 
CHANG, A.C.; GRANATO, T.C. \& PAGE, A.L. A methodology for establishing phytotoxicity criteria for chromium, copper, nickel, and zinc in agricultural land application of municipal sewage sludges. J . Environ. Qual., 21:521-536, 1992.

DANG, Y.P.; CHABRA, R. \& VERMA, K.S. Effect of Cd, Ni, Pb and $\mathrm{Zn}$ on growth and chemical composition of onions and fenugreek. Comm. Soil Sci. Plant Anal., 21:717-735, 1990.

DAY, P.R. Particle fractionation and particle-size analysis. In: BLACK, C.A., ed. Methods of soil analysis: part 1. Madison, American Society of Agronomy, 1965. p.545-567.

EMPRESA BRASILEIRA DE PESQUISA AGROPECUÁRIA EMBRAPA. Centro Nacional de Pesquisas de Sol os. Manual de métodos de análise de solo. 2.ed. Rio de J aneiro, 1997. 212p.

HAQ, A.U. \& MILLER, M.H. Prediction of available soil Zn, $\mathrm{Cu}$, and $\mathrm{Mn}$ using chemical extractants. Agron. J ., 64:779782, 1972.

KORCAK, R.F. \& FANNING, D.S. Extractability of cadmium, copper, nickel, and zinc by double acid versus DTPA and plant content at excessive soil levels. J. Environ. Qual., 7:506-512, 1978

LI, Z. \& SHUMAN, L.M. Mehlich-1 and DTPA-Extractable lead in soils in relation to properties. Comm. Soil Sci. Plant Anal., 28:351-363, 1997.

LINDSAY, W.L. \& NORVELL, W.A. Development of a DTPA soil test for zinc, iron, manganese, and copper. Soil Sci. Soc. Am. J ., 42:421-428, 1978.

LUND, Z.F. \& DOSS, B.D. Residual effects of dairy cattle manure on plant growth and soil properties. Agron. J., 72:123-130, 1980.

MacLEAN, A.J .; HALSTEAD, R.L. \& FINN, B.J . Extractability of added lead in soils and its concentration in plants. Can. J. Soil Sci., 49:327-334, 1969.

MCLAREN, R.G. \& CRAWFORD, D.V. Studies on soil copper. I. The fractionation of copper in soils. J. Soil Sci., 24:172181, 1973.

MEHLICH, A. Mehlich-3 soil test extractant: a modification of Mehlich-2 extractant. Comm. Soil Sci. Plant Anal., 15:1409$1416,1984$.

MULCHI, C.L.; ADAMU, C.A.; BELL, P.F. \& CHANEY, R.L. Residual heavy metal concentrations in sludge-amended coastal plain soils - I. Comparison of extractants. Comm. Soil Sci. Plant Anal., 22:919-41, 1991.

MULLINS, G.L.; MARTENS, D.C.; MILLER, W.P.; KORNEGAY, E.T. \& HALLOCK, D.L. Copper availability, form, and mobility in soils from three annual copper-enriched hog manure applications. J . Environ. Qual., 11:316-320, 1982.
OLIVEIRA, M.F.G.; NOVAIS, R.F.; NEVES, J.C.L.; VASCONCELLOS, C.A. \& ALVES, M. Relação entre o zinco "disponível", por diferentes extratores, e as frações de zinco em amostras de solos. R. Bras. Ci. Solo, 23:827836,1999 .

RAIJ , B. van, QUAGGIO, J .A.; CANTARELA, H.; FERREIRA, M.E.; LOPES, A.S. \& BATAGLIA, O.C. Análise química do solo para fins defertilidade. Campinas, Fundação Cargill, 1987. 170p.

RITCHEY, K.D.; COX, F.R.; GALRÃO, E.Z. \& YOST, R.S. Disponibilidade de zinco para as culturas do milho, sorgo e soja em Latossolo Vermel ho-Escuro argiloso. Pesq. Agropec. Bras. 21:215-225, 1986

SANDERS, J.R.; ADAMS, T.M. \& CHRISTENSEN, B.T. Extractability and bioavailability of zinc, nickel, cadmium and copper in three Danish Soils sampled 5 years after application of sewage sludge. J . Sci. Food Agric., 37:11551164,1986

SAS INSTITUTE. Statistical Analysis System Institute. SAS/ STAT U ser's guide, version 6, 4.ed. Cary, 1989. v.1/2. 1686p.

SHUMAN, L.M. Effect of liming on the distribution of manganese, copper, iron, and zinc among soil fractions. Soil Sci. Soc. Am. J ., 50:1236-1240, 1986.

SHUMAN, L.M. Effect of organic matter on the distribution of manganese, copper, iron, and zinc in soil fractions. Soil Sci., 146:192-198, 1988.

SHUMAN, L.M. Effect of organic waste amendments on cadmium and lead in soil fractions of two soils. Comm. Soil Sci. Plant Anal., 29:2939-2952, 1998.

SILVA, F.C.; BOARETTO, A.E.; BERTON, R.S.; ZOTELLI, H.B.; PEXE, C.A. \& MENDONÇA, E. Cana-de-açúcar cultivada em solo adubado com lodo de esgoto: nutrientes, metais pesados e produtividade. Pesq. Agropec. Bras., 33:1-8, 1998.

TEDESCO, M.J .; GIANELLO, C.; BISSANI, C.A.; BOHNEN, H. \& VOLKWEISS, S.J . Análise de solos, plantas e outros materiais. 2.ed. Porto Alegre, Universidade Federal do Rio Grande do Sul, 1995.174p. (Boletim Técnico, 5)

WALLACE, G.A. \& WALLACE, A. Lead and other potentially toxic heavy metals in soil. Comm. Soil Sci. Plant Anal., 25:137-141, 1994.

WEAR, J I. \& EVANS, C.E. Relationship of zinc uptake by corn and sorghum to soil zinc measured by three extractants. Soil Sci. Soc. Am. Proc., 32:543-546, 1968.

WEAR, J.I. \& SOMMER, A.L. Acid-extractable zinc of soils in relation to the occurrence of zinc deficiency symptoms of corn: A method of analysis. Soil Sci. Soc. Am. Proc., 12:143$144,1947$. 